\title{
Prediction of late-onset fetal growth restriction using a combined first- and second-trimester screening model in South Chinese infants: a retrospective study
}

\section{Haiqing Zheng}

Guangzhou Women and Children's Medical Center

Yan Feng

Guangzhou Women and Children's Medical Center

Jiexin Zhang

Guangzhou Women and Children's Medical Center

Kuanrong Li

Guangzhou Women and Children's Medical Center

Huiying Liang

Guangzhou Women and Children's Medical Center

\section{Shanshan Mei}

Guangzhou Women and Children's Medical Center

\section{Dajun Fang}

Guangzhou Women and Children's Medical Center

Qiuming He

Guangzhou Women and Children's Medical Center

\section{Suting Xu}

Guangzhou Women and Children's Medical Center

Guanglan Zhang ( $\sim$ gnandqj@126.com )

Guangzhou Women and Children's Medical Center https://orcid.org/0000-0002-5689-7860

\section{Wei Zhong}

Guangzhou Women and Children's Medical Center

\section{Research article}

Keywords: Late-onset fetal growth restriction, Prediction, South Chinese infants, First- and secondtrimester screening

Posted Date: November 27th, 2019 
DOI: https://doi.org/10.21203/rs.2.17844/v1

License: (c) (1) This work is licensed under a Creative Commons Attribution 4.0 International License. Read Full License 


\section{Abstract}

Background Prediction models for early and late fetal growth restriction (FGR) have been established in many high-income countries. However, prediction models for late FGR in China are limited. This study aimed to develop a simple combined first- and second-trimester prediction model for screening late-onset FGR in South Chinese infants. Methods This retrospective study included 2258 women who had singleton pregnancies and received routine ultrasound scans. Late-onset FGR was defined as a birth weight $<$ the 10th percentile plus abnormal Doppler indices and/or a birth weight below the 3rd percentile after 32 weeks, regardless of the Doppler status. Multivariate logistic regression was used to develop a prediction model.Results Ninety-three fetuses were identified as late-onset FGR. The significant predictors for lateonset FGR were maternal age, height, weight, and medical history; the second-trimester head circumference $(\mathrm{HC}) /$ abdominal circumference $(\mathrm{AC})$ ratio; and the estimated fetal weight (EFW). This model achieved a detection rate (DR) of $52.6 \%$ for late-onset FGR at a $10 \%$ false positive rate (FPR) (area under the curve (AUC): $0.80,95 \% \mathrm{Cl} 0.76-0.85$ ). Conclusions A multivariate model combining first- and second-trimester default tests can detect $52.6 \%$ of cases of late-onset FGR. Further studies with more screening markers are needed to improve the detection rate.

\section{Background}

Fetal growth restriction (FGR) is a pathological condition that is mainly associated with placental insufficiency[1]. It is associated with an increased risk of perinatal mortality and morbidity and is felt to be as high as $5.5 \%$ in certain population-based studies [2-4]. A major focus of prenatal care is to determine whether a fetus is at risk for growth restriction and to identify growth-restricted fetuses. Research has shown that the antenatal identification of FGR results in reductions in mortality and morbidity. A large study, which included over 92,000 singletons, found lower stillbirth fetal rates for antenatally detected FGR (9.7\%o) than for undetected FGR (18.9\%o)[5]. However, the inaccuracy of early detection remains unresolved. Because the degree of placental disease in late FGR is mild, the umbilical Doppler index is normal in virtually all cases [6]. Late-onset FGR represents $70-80 \%$ of FGR cases [7]. Early detection could improve the outcomes of these fetuses by establishing follow-up intervals and optimal delivery timing[8]. Unfortunately, late-onset FGR often goes undetected[5], thereby leading to higher rates of cesarean-section delivery due to fetal distress, neonatal acidosis, and admission to the neonatal intensive care unit[9].

Previous studies have assessed the performance of prenatal screening, with the combination of maternal characteristics, fetal biometry, Doppler parameters and biochemical markers, for the detection of FGR[10-14]. Although they showed improved performance in the identification of early onset FGR, these models remained unsatisfactory for late-onset FGR. Furthermore, these previously reported models were complex and were thus difficult to apply in the real-world clinical setting. Therefore, the aim of this study was to develop a simple screening model for the late-onset FGR of Chinese infants using routine screening markers. 


\section{Methods \\ Participants}

This retrospective observational study included women who had a singleton pregnancy, had received routine prenatal care during their first, second and third trimesters and had delivered a baby after 32 weeks at Guangzhou Women and Children's Medical Center between January 2013 and December 2016. Gestational age (GA) was calculated by crown-rump length (CRL). Study participants were excluded due to the following patient variables: the presence of structural defects that were suspected at the time of routine scans and/or confirmed postnatally; termination of pregnancy, intrauterine death and stillbirth before 32 weeks; no knowledge of the first day of the last menstruation period (LMP) or the lack of a regular menstrual cycle duration of 28 days plus or minus 4 days; and gestation that was inconsistent with the findings of an ultrasound examination at 6-7 weeks. During the initial database search, 2478 women met the inclusion criteria. Of these women, 220 met either the exclusion criteria or had incomplete data for recorded factors, leaving 2258 women for the analysis. The Medical Ethics Committee of Guangzhou Women and Children's Medical Center approved this study.

\section{Maternal characteristics and clinical variables}

Data on maternal characteristics were collected retrospectively from the medical records and included maternal age, pregestational maternal height and weight, nulliparity (no previous deliveries after 24 weeks gestation), conception method (spontaneous or via an assisted reproductive technique), maternal medical history (chronic hypertension, diabetes mellitus, renal disease, autoimmune disease or coagulation disorders), and obstetrical history (e.g., previous stillbirth, miscarriage or fetal anomaly). Within the study, the main clinical variable was maternal blood pressure (BP), which was measured at the time of the first trimester ultrasound $\left(11^{+0}\right.$ to $13^{+6}$ weeks of gestation) with an automatic blood pressure monitor (OMRON HBP-9020, Kyoto, Japan). BP was measured with the woman comfortably seated after a 5-minute rest. The mean arterial pressure (MAP) was calculated as diastolic BP + (systolic BP-diastolic $\mathrm{BP}) / 3$.

\section{Maternal blood biomarkers}

Maternal serum free- $\beta$-human chorionic gonadotropin (HCG) and pregnancy-associated plasma protein $A$ (PAPP-A) levels were measured at the time of the nuchal translucency scan $\left(11^{+0}\right.$ to $13^{+6}$ weeks). The measured concentrations of the two hormones were converted to the multiple of the maternal-weightadjusted gestation-specific median for the local Chinese population, followed by $\log _{10}$ transformation $\left(\log _{10}\right.$ PAPP-A ${ }_{M o M}$ and $\log _{10} f \beta-h C G_{M o M}$, respectively[15]. These concentrations were measured using a time-resolved 1234 Delfia ${ }^{\circledR}$ (Wallac, Turku, Finland). 


\section{Doppler measurements}

Transabdominal ultrasound with Doppler evaluation was performed during pregnancy using a Voluson Expert E8 (GE Healthcare), using curvilinear 2.0 to $5.0 \mathrm{MHz}$ transducers. CRL was measured in a true midsagittal plane with the genital tubercle and the fetal spine longitudinally in view, in the GA range of $11^{+0}$ weeks to $13^{+6}$ weeks. In the second trimester (24-28 weeks), the biparietal diameter, head circumference, abdominal circumference and femur length were measured. The second-trimester estimated fetal weight (EFW) was calculated based on the Hadlock formula[16]. The EFW percentile was calculated using local standards [17]. The umbilical artery-pulsatility index (UA-PI) was calculated from a free-floating portion of the umbilical cord. The middle cerebral artery (MCA) was measured at the axial view of the fetal head, in the inner one-third of its course to the circle of Willis.

\section{Fetal growth restriction}

FGR was defined as a birth weight < the 10th percentile for GA with abnormal Doppler indices (either UA$\mathrm{PI}>$ the 95th percentile or MCA PI < the 5th percentile for gestational age)[18], and/or as a birth weight of less than the 3rd percentile[19] according to local standards, regardless of the Doppler status before delivery. Late-onset FGR was defined as FGR that was newly diagnosed at greater than 32 weeks gestation[20].

\section{Statistical analysis}

Continuous variables were analyzed using the unpaired Student's t-test, while categorical variables were analyzed using the Pearson $\chi^{2}$ test. The Z-score was calculated by dividing the difference between the observed value and the gestational age-specific mean with the standard deviation (SD). The observed measurements of CRL, MAP, UA-PI, HC/AC and EFW were expressed as the respective Z-scores corrected for gestational age. If one woman had repeated test results in one trimester, then the mean Z-score was calculated. Multivariate logistic regression analysis with backward stepwise elimination was used to determine which maternal factors and aspects of the obstetrical history significantly contributed to predicting late-onset FGR, and accuracy was assessed by receiver-operating characteristic (ROC) curve. The predicted probabilities from each regression model were documented, and the detection rates (DRs) for a $10 \%$ false positive rate (FPR) were calculated. The statistical software package $\mathrm{R}$ version 3.4.1 was used for all data analyses.

\section{Results}

\section{Characteristics and outcomes between the late-onset FGR group and normal growth group}


Of the 2258 neonates, 93 (4.12\%) met the criteria for late-onset FGR. Table 1 shows the characteristics for late-onset FGR and the characteristics of nongrowth restricted neonates. The maternal age, height and weight were lower in late-onset FGR neonates than in those exhibiting normal growth. There were no significant differences with respect to gravidity, maternal medical history, previous stillbirth or obstetrical history. No study participant reported tobacco use or alcohol use. The EFW Z-score and HC/AC Z-score were significantly lower and higher, respectively, for neonates with late-onset FGR than for those showing normal growth. The two groups differed in their rates of gestational diabetes mellitus and birth weight. 
Table 1

Maternal characteristics, biophysical and biochemical predictive variables and outcomes of the participants subdivided into late-onset FGR and normal growth

\begin{tabular}{|c|c|c|c|}
\hline Maternal characteristics & Late-onset FGR $(n=93)$ & Normal growth $(n=2165)$ & $\mathbf{P}$ \\
\hline Age (years) & $31.13 \pm 3.82$ & $29.83 \pm 3.91$ & 0.001 \\
\hline Height (cm) & $158.49 \pm 4.89$ & $160.21 \pm 4.88$ & 0.001 \\
\hline Weight (kg) & $53.44 \pm 4.49$ & $56.23 \pm 4.43$ & 0.002 \\
\hline Assisted reproductive technology & $1(1.1)$ & $38(1.8)$ & 0.931 \\
\hline Nulliparous & $69(74.2 \%)$ & $1450(67.0 \%)$ & 0.146 \\
\hline Maternal medical history & $8(8.6 \%)$ & $111(5.1 \%)$ & 0.142 \\
\hline Previous stillbirth & $1(1.1 \%)$ & $6(0.3 \%)$ & $0.175^{\mathrm{a}}$ \\
\hline Adverse obstetrical history & $3(3.2 \%)$ & $44(2.0 \%)$ & 0.430 \\
\hline \multicolumn{4}{|c|}{ Biophysical and biochemical predictive variables } \\
\hline \multicolumn{4}{|c|}{ First-trimester parameters (11-13 weeks) } \\
\hline Hemoglobin level & $118.46 \pm 10.34$ & $117.69 \pm 10.26$ & 0.415 \\
\hline PAPP-A (MoM) & $1.23 \pm 0.68$ & $1.31 \pm 0.72$ & 0.295 \\
\hline$\beta H C G(M o M)$ & $1.44 \pm 0.93$ & $1.52 \pm 1.03$ & 0.432 \\
\hline MAP (Z-score) & $-0.06 \pm 1.17$ & $-0.09 \pm 1.04$ & 0.796 \\
\hline \multicolumn{4}{|c|}{ Second-trimester parameters (24-28 weeks) } \\
\hline EFW (Z-score) & $-1.02 \pm 0.87$ & $-0.08 \pm 1.05$ & $<0.001$ \\
\hline HC/AC ratio (Z-score) & $0.56 \pm 0.90$ & $-0.01 \pm 1.06$ & $<0.001$ \\
\hline UA-PI (Z-score) & $0.03 \pm 1.24$ & $-0.03 \pm 0.95$ & $<0.616$ \\
\hline \multicolumn{4}{|l|}{ Maternal and neonatal outcomes } \\
\hline Preeclampsia & $2(2.2 \%)$ & $25(1.2 \%)$ & 0.369 \\
\hline Gestational diabetes mellitus & $10(10.8 \%)$ & $406(18.8 \%)$ & 0.051 \\
\hline Gestational age at delivery & $38.63 \pm 1.53$ & $38.88 \pm 4.63$ & 0.661 \\
\hline
\end{tabular}

“a”, the P value was obtained by Pearson's chi-squared test with Yates's continuity correction;

FGR, fetal growth restriction; MoM, multiple of the median; PAPP-A, pregnancy-associated plasma protein $A ; H C G$, human chorionic gonadotropin; MAP, mean arterial pressure; EFW, estimated fetal weight; $\mathrm{HC}$, head circumference; $\mathrm{AC}$, abdominal circumference; UA-PI, umbilical artery pulsatility index. 


\begin{tabular}{|llll|}
\hline Maternal characteristics & Late-onset FGR $(\mathbf{n = 9 3 )}$ & Normal growth $(\mathbf{n = 2 1 6 5 )}$ & $\mathbf{P}$ \\
\hline Cesarean delivery & $32(34.4 \%)$ & $821(37.9 \%)$ & 0.493 \\
\hline Birth weight (grams) & $2321.71 \pm 331.80$ & $3216.22 \pm 418.84$ & $<0.001$ \\
\hline 5-min Apgar score & $9.06 \pm 0.25$ & $9.10 \pm 0.41$ & 0.303 \\
\hline “a”, the P value was obtained by Pearson's chi-squared test with Yates's continuity correction; & \\
\hline $\begin{array}{l}\text { FGR, fetal growth restriction; MoM, multiple of the median; PAPP-A, pregnancy-associated plasma } \\
\text { protein A; HCG, human chorionic gonadotropin; MAP, mean arterial pressure; EFW, estimated fetal } \\
\text { weight; HC, head circumference; AC, abdominal circumference; UA-PI, umbilical artery pulsatility index. }\end{array}$ \\
\hline
\end{tabular}

\section{Predicting late-onset FGR}

The risk factors for late-onset FGR were calculated using multivariate logistic regression analysis. The regression coefficients and adjusted odds ratios of each contributor are presented in Table 2. After screening, the significant independent factors in the prediction of late-onset FGR were maternal age (OR $0.93,95 \% \mathrm{Cl} 0.87-0.98)$, maternal height (OR 0.96, 0.91-1.0), maternal weight (OR 0.97, 95\% Cl 0.95-1.00), maternal medical history (OR $2.0295 \% \mathrm{Cl} 0.86-4.19)$, the second-trimester $\mathrm{HC} / \mathrm{AC}$ ratio Z-score (OR 1.46, $95 \% \mathrm{Cl} 1.22-1.81$ ) and the EFW Z-score (OR $0.45,95 \% \mathrm{Cl} 0.36-0.56)$. The area under the ROC curve for this model was 0.80 ( $95 \% \mathrm{Cl}: 0.76-0.85)$. At a $10 \%$ false positive rate (FPR), this model predicted $52.6 \%$ of cases of late-onset FGR (Table 3, Fig. 1).

Table 2

Backward stepwise logistic regression model for the prediction of late-onset FGR

\begin{tabular}{|llllll|}
\hline Factors & Coefficient & SE & OR & $95 \% \mathrm{Cl}$ & P \\
\hline Maternal medical history & 0.71 & 0.39 & 2.02 & $0.86-4.19$ & 0.060 \\
\hline Maternal age & -0.07 & 0.03 & 0.93 & $0.87-0.98$ & 0.011 \\
\hline Maternal height & -0.04 & 0.02 & 0.96 & $0.91-1.00$ & 0.079 \\
\hline Maternal weight & -0.03 & 0.01 & 0.97 & $0.95-1.00$ & 0.069 \\
\hline EFW Z-score & -0.79 & 0.12 & 0.45 & $0.36-0.56$ & $<0.001$ \\
\hline HC/AC Z-score & 0.38 & 0.10 & 1.46 & $1.22-1.81$ & $<0.001$ \\
\hline $\begin{array}{l}\text { SE, standard error, OR, odds ratio; Cl, confidence interval; FGR, fetal growth restriction; HC, head } \\
\text { circumference; AC, abdominal circumference; EFW, estimated fetal weight. }\end{array}$ & \\
\hline $\begin{array}{l}\text { * Maternal medical history included chronic hypertension, diabetes mellitus, renal disease, } \\
\text { autoimmune disease and coagulation disorders. }\end{array}$ & \\
\hline
\end{tabular}


Table 3

Predictive values of screening for late-onset FGR

\begin{tabular}{|llll|}
\hline Screening test & AUC & $95 \% \mathrm{Cl}$ & DR $(95 \% \mathrm{Cl})$ at $10 \% \mathrm{FPR}$ \\
\hline Maternal factors & 0.64 & $0.58-0.69$ & $23.6(15.0-32.2)$ \\
\hline +HC/AC ratio & 0.71 & $0.65-0.76$ & $30.1(21.5-39.7)$ \\
+HC/AC ratio + EFW & 0.80 & $0.76-0.85$ & $52.6(43.0-63.4)$ \\
\hline
\end{tabular}

FGR, fetal growth restriction; AUC: area under the curve; DR: detection rate; FPR, false positive rate; HC, head circumference; AC, abdominal circumference; EFW, estimated fetal weight.

The following model best fits the prediction for late-onset FGR (risk $=\mathrm{e}^{\mathrm{y}} /\left(1+\mathrm{e}^{\mathrm{y}}\right)$ :

Logit $(p)=7.29-0.07 *$ maternal age- $0.04 *$ maternal height $-0.03^{\star}$ maternal weight $+0.71 *$ maternal medical history $+0.38 * \mathrm{HC} / \mathrm{AC}$ Z-score-0.79* EFW Z-score

\section{Discussion}

Late-onset FGR is easy to miss clinically because of its insidious onset and mild symptoms. This study showed that a combined model, including maternal characteristics and second-trimester ultrasonographic parameters, was able to predict $52.6 \%$ of cases of late-onset FGR. On the other hand, we found no associations among assisted conception, previous stillbirth, an adverse obstetrical history, the hemoglobin level and late-onset FGR, which was inconsistent with the findings of other studies [10, 11, 21].

The detection rate for late-onset FGR was $52.6 \%$, which is slightly lower than the DR of $59.6 \%$ reported by A. Sotiriadis et al[10] and the DR of $64 \%$ reported by J. Miranda et al[13] when using maternal characteristics and third-trimester EFW percentiles. F. Crovetto et al[14] used a combination of maternal risk factors, the first-trimester MAP, the mean UtA-PI and the soluble Fms-like tyrosine kinase-1 (sFlt1)/placental growth factor (PLGF) ratio and detected $66 \%$ of cases of late-onset FGR, at a $10 \%$ FPR. These combined models were capable of detecting late-onset FGR more frequently or earlier. However, the application of UtA-PI, PLGF and sFIt-1 may be clinically difficult since these tests are not a component of routine prenatal care. The combined use of maternal factors and second-trimester markers in our study could achieve similar outcomes, and the data were from the default test for chromosomal abnormalities and fetal growth monitoring, making the model simpler, cheaper and easier to apply in local hospitals.

Additionally, it is noteworthy that the addition of the EFW Z-score increased the DR from $30.1-52.6 \%$, highlighting the importance of the EFW in the prediction of late-onset FGR. Similarly, a recent study that included 30,849 singleton pregnancies found that a screening approach that combined the maternal characteristics and history with the EFW Z-score increased the DR from 30-80\% for SGA neonates that were delivered within $<5$ weeks of assessment, with a FPR of $10 \%[22]$. The third-trimester customized 
estimated fetal weight percentiles could moderately predict FGR[23]. The EFW is objectively measured and not affected by ethnicity, so it is necessary to define its role in the clinical management of the prediction of late-onset FGR in pregnancy. However, some women are not routinely scanned in late pregnancy but are selected for third-trimester ultrasonography based on prepregnancy factors and serial measurements of the symphyseal-fundal height $[24,25]$. This approach identifies only a few SGA babies, whereas a large proportion of pregnant women who are at increased risk for delivering babies with lateonset FGR cannot be detected. Thus, it is important to routinely monitor fetal growth by ultrasonic examination during pregnancy.

The strength of the study is the large number of pregnant women receiving routine care in a well-defined gestational-age range that is widely used for screening chromosomal defects and for assessments of fetal anatomy and growth. The screening markers were default tests from routine care, which makes the model very convenient and cheap. In China and other countries with inadequate medical resources, this model can significantly save time and reduce economic costs.

However, we acknowledge that there were some limitations in our study. First, given the nature of the retrospective design, data pertaining to many meaningful parameters, such as previous history of FGR, UtA-PI, PLGF and sFIt-1, which have high contributions to the prediction of FGR[13, 14, 26], were unavailable in the present study; otherwise, the detection rate would have been higher. Thus, we attempt to add the test of UtA-PI in pregnant women from the first trimester throughout the third trimester to further improve the detection rate of late FGR. In addition, nearly all participants reported no tobacco use or alcohol use, preventing us from controlling for differences in smoking or alcohol use.

\section{Conclusion}

Our study demonstrated that a combination of first- and second-trimester default test results detected $52.6 \%$ of cases of late-onset FGR. This retrospective study provides a simple model for the prediction of late-onset FGR, and further studies may be needed to confirm these results using a prospective design.

\section{Abbreviations}

FGR, fetal growth restriction; $C R L$, crown-rump length; $H C$, head circumference; $A C$, abdominal circumference; $E F W$, estimated fetal weight; DR, detection rate; FPR, false positive rate; AUC, area under the curve; SGA, small-for-gestational-age; GA, gestational age; LMP, last menstruation period; BP, blood pressure; MAP, mean arterial pressure; HCG, human chorionic gonadotropin; PAPP-A, pregnancyassociated plasma protein A; UA-PI, Umbilical artery-pulsatility index; UtA-PI, uterine artery-pulsatility index; MCA, middle cerebral artery; SD, standard deviation; ROC, received operating characteristic; OR, odds ratio; sFlt-1, soluble Fms-like tyrosine kinase-1; PLGF, placental growth factor

\section{Declarations}


Competing interests

The authors declare that they have no competing interests.

\section{Funding}

This study was funded by the Guangzhou Institute of Pediatrics/Guangzhou Women and Children's Medical Center (Funding number: YIP-2019-055).

Authors' contributions

HL, GZ and WZ conceived and designed the study. DF, SX and JZ carried out the data collection and interpreted the data. $\mathrm{HZ}$ and $\mathrm{KL}$ performed the data analyses. SM and DF helped perform the analysis with constructive discussions. SM, QH, GZ and WZ contributed to the interpretation of the results. $\mathrm{HZ}$ and YF wrote the first draft. KL, GZ and WZ revised the manuscript for important intellectual content. All authors reviewed drafts and approved the manuscript.

Acknowledgements

We thank Timothy C. Lee from Texas Children's Hospital, Houston, Texas, USA, for the language revision.

\section{References}

1.

Lackman F, Capewell V, Gagnon R, Richardson B. Fetal umbilical cord oxygen values and birth to placental weight ratio in relation to size at birth. Am J Obstet Gynecol. 2001;185(3):674-82.

2.

McIntire DD, Bloom SL, Casey BM, Leveno KJ. Birth weight in relation to morbidity and mortality among newborn infants. N Engl J Med. 1999;340(16):1234-8.

3.

Garite TJ, Clark R, Thorp JA. Intrauterine growth restriction increases morbidity and mortality among premature neonates. Am J Obstet Gynecol. 2004;191(2):481-7.

4.

Lees C, Marlow N, Arabin B, Bilardo CM, Brezinka C, Derks JB, et al. Perinatal morbidity and mortality in early-onset fetal growth restriction: cohort outcomes of the trial of randomized umbilical and fetal flow in Europe (TRUFFLE). Ultrasound Obstet Gynecol. 2013;42(4):400-8.

5.

Gardosi J, Madurasinghe V, Williams M, Malik A, Francis A. Maternal and fetal risk factors for stillbirth: population based study. BMJ. 2013;346:f108.

6.

Oros D, Figueras F, Cruz-Martinez R, Meler E, Munmany M, Gratacos E. Longitudinal changes in uterine, umbilical and fetal cerebral Doppler indices in late-onset small-for-gestational age fetuses. Ultrasound Obstet Gynecol. 2011;37(2):191-5.

7.

Unterscheider J, Daly S, Geary MP, Kennelly MM, McAuliffe FM, O'Donoghue K, et al. Optimizing the definition of intrauterine growth restriction: the multicenter prospective PORTO Study. Am J Obstet Gynecol. 2013;208(4):290 e1-6. 
Figures

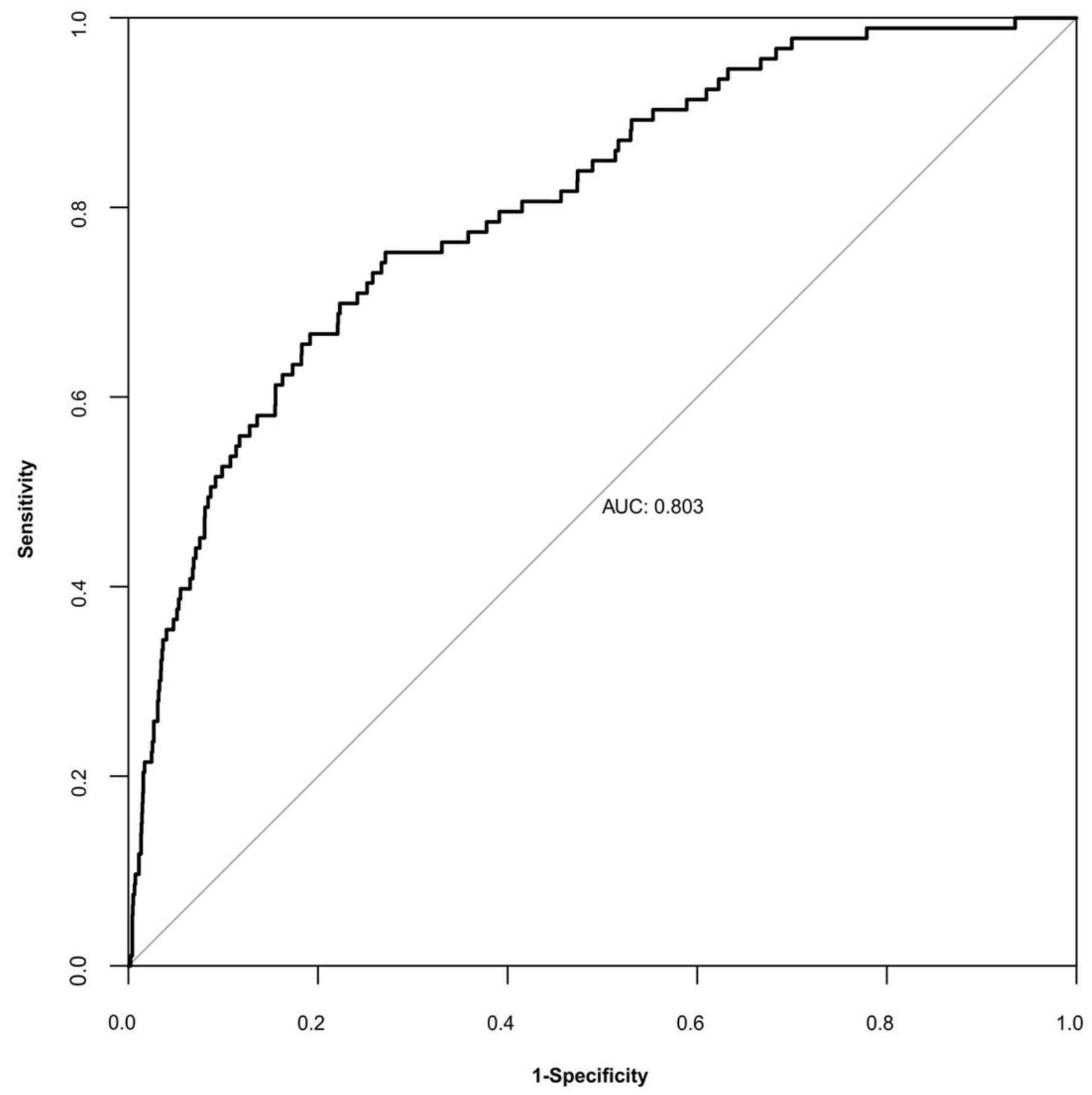

Figure 1

ROC curve for the prediction of late fetal growth restriction with the combination of maternal characteristics (maternal age, maternal height, maternal weight and maternal medical history) and ultrasound data (second-trimester head circumference/abdomen circumference ratio and estimated fetal weight). 